\title{
THE ZETA FUNCTION OF TORAL ENDOMORPHISMS
}

\author{
JAMES W. ENGLAND ${ }^{1}$
}

\begin{abstract}
In this note we give a completely elementary way to compute the number of fixed points of a certain class of toral endomorphisms. This, in turn, gives the zeta function of these endomorphisms.
\end{abstract}

We first review the small collection of facts needed to understand the theorem. We denote by $\boldsymbol{R}^{n}$ the group of $n$-tuples of real numbers and by $Z^{n}$ the subgroup of $R^{n}$ of integral lattice points. The $n$-torus may be thought of as the group obtained by factoring $Z^{n}$ out of $\boldsymbol{R}^{n}$, i.e. $\boldsymbol{R}^{n} / Z^{n}$. If $A$ is an $n \times n$ matrix with all integer entries then, since $A$ leaves $Z^{n}$ invariant, it induces an endomorphism $T_{A}$ on $R^{n} / Z^{n}$ by $T_{A}\left(x+Z^{n}\right)=$ $A x+Z^{n}$. It can be shown that if $T$ is any endomorphism of $R^{n} / Z^{n}$ then there exists a matrix $A$ with all integer entries such that $T=T_{A}$. It is easy to see that if $T_{A}^{k}=T_{A} \cdots T_{A}$, $k$ terms, then $T_{A}^{k}=T_{A^{k}}$. The class of endomorphisms we wish to consider are those whose corresponding matrices have no eigenvalues equal to a root of unity. In case $\operatorname{det} A= \pm 1$ then these are exactly the ergodic automorphisms [1].

THEOREM. Let $A$ be a $n \times n$ integral entry matrix with the property that it has no eigenvalues equal to a root of unity. Let $T_{A}$ denote the endomorphism that $A$ induces on the $n$-torus, $\boldsymbol{R}^{n} / Z^{n}$. If $N_{k}$ denotes the number of fixed points of $T_{A}^{k}=T_{A^{k}}$ then $N_{k}=\left|\operatorname{det}\left(A^{k}-I\right)\right|$ for $k=1,2,3, \cdots$.

Proof. For $\tilde{x} \in \boldsymbol{R}^{n} / Z^{n}$ we write $\tilde{x}=x+Z^{n}$ with $x=\left(x_{1}, \cdots, x_{n}\right)$, $0 \leqq x_{i}<1, i=1,2, \cdots, n$. Let $k$, a positive integer, be given. Now, $\tilde{x}$ is fixed under $T_{A^{k}}$ if and only if $A^{k} x=x \bmod \left(Z^{n}\right)$, or, if and only if $\left(A^{k}-I\right) x \in Z^{n}$. Since $A^{k}$ has no eigenvalues equal to 1 it follows that $A^{k}-I$ is one-to-one. Thus there is a one-to-one correspondence between these points $x$ of the half open unit cube which have the property that $\left(A^{k}-I\right) x \in Z^{n}$, and the integral lattice points contained in the image of the half open unit cube under $A^{k}-I$. Considering the volume interpretation of determinates it is geometrically clear that this number is exactly

Received by the editors August 31, 1971.

AMS 1970 subject classifications. Primary 58F20; Secondary 28A65, 54H20.

Key words and phrases. Fixed points, matrix, eigenvalues, ergodic automorphisms.

1 Supported in part by NSF Grant GP-19103. 
$\left|\operatorname{det}\left(A^{k}-I\right)\right|$. A rather dull proof of this fact can be seen as follows. If we let $\left(A^{k}-I\right) \mid Z^{n}$ denote the restriction of $\left(A^{k}-I\right)$ to $Z^{n}$, then the number of integral lattice points in the image of the half open unit cube under $A^{k}-I$ is equal to the order of the group $Z^{n} / \operatorname{Im}\left[\left(A^{k}-I\right) \mid Z^{n}\right]$. Now, if $p=\left|\operatorname{det}\left(A^{k}-I\right)\right|$ then $p$ is an integer and for $x \in Z^{n}, x \in \operatorname{Im}\left[\left(A^{k}-I\right) \mid Z^{n}\right]$ if and only if $x=p y$ for some $y \in Z^{n}$. This proves the theorem.

Recall from [2, p. 764] that if $f: X \rightarrow X$ is continuous and if $N_{k}$ equals the number of isolated fixed points of $f^{k}$ and if $N_{k}<\infty$ for all $k$ then the Artin-Mazur zeta function of $f$ is defined to be

$$
\zeta_{f}(z)=\exp \left(\sum_{k=1}^{\infty} \frac{N_{k}}{k} z^{k}\right) .
$$

The above theorem gives the zeta function for all toral endomorphisms for which $N_{k}<\infty$ for all $k$, that is, for all toral endomorphisms which have no eigenvalues equal to a root of unity. The result in this case is the same as the result in the case when $A$ has no eigenvalues on the unit circle which is given in [2, p. 769].

Peter Walters has recently shown me that, independently, he has obtained the same proof.

\section{BIBLIOGRAPHY}

1. Paul R. Halmos, Lectures on ergodic theory, Publ. Math. Soc. Japan, no. 3, Math. Soc. Japan, Tokyo, 1956. MR 20 \#3958.

2. S. Smale, Differentiable dynamical systems, Bull. Amer. Math. Soc. 73 (1967), 747-817. MR 37 \#3598.

Department of Mathematics, Swarthmore College, Swarthmore, Pennsylvania 19081 